\title{
Measurement of Critical Energy for Direct Initiation of Spherical Detonations in Stoichiometric High-Pressure $\mathrm{H}_{2}-\mathrm{O}_{2}$ Mixtures
}

\author{
Vsevolods Kamenskihss ${ }^{1}$, Hoi Dick $\mathrm{Ng}^{2 \dagger}$ and John H.S. Lee ${ }^{1}$ \\ ${ }^{1}$ Department of Mechanical Engineering, McGill University \\ Montréal, Québec, Canada \\ ${ }^{2}$ Department of Mechanical and Industrial Engineering, Concordia University \\ Montréal, Québec, Canada \\ ${ }^{\dagger}$ Corresponding author \\ Department of Mechanical and Industrial Engineering \\ Concordia University \\ 1455 de Maisonneuve Blvd. West \\ Montréal, Québec, H3G 1M8, Canada \\ E-mail : hoing@encs.concordia.ca \\ Tel: 1 (514) 848-2424 (ext. 3177) \\ Fax: 1 (514) 848-3175
}

Revised manuscript submitted to Combustion and Flame

February 17, 2010 


\begin{abstract}
In this study, the critical energy for direct initiation of spherical detonations in stoichiometric high-pressure hydrogen-oxygen mixtures are measured and investigated to look at the effect of explosion limits on the detonation sensitivity. Results up to an initial pressure of 20 atm are obtained. Experiments are carried out in a spherical bomb and direct initiation is achieved via spark ignition from a high voltage capacitor discharge. A detailed description of different methods to obtain a good estimate of the correct amount of energy deposited into the mixture used to initiate the detonation, including the calorimeter method and current method, is provided. It is demonstrated that at elevated initial pressure, the second explosion limit effect plays a significant role leading to slow branching reactions and the detonation sensitivity of hydrogen mixtures is comparable to other common hydrocarbon mixtures at such condition.
\end{abstract}

Keywords: Detonation sensitivity; direct initiation; critical energy; high-pressure; spark ignition 


\section{INTRODUCTION}

The critical energy for direct initiation of detonation, i.e. an "instantaneous" formation of a detonation in the decay of a strong blast wave ensued from a powerful ignition source [1], is an important dynamic parameter to provide a quantitative measurement of the detonation sensitivity of an explosive mixture in an unconfined environment [2]. Direct initiation of detonations and measurement of critical energies in hydrogen-oxygen and hydrogen-air mixtures had been investigated in the past (e.g. [2-7]). However, most of these studies only carried the experiments and measured the critical energy at low (or sub-atmospheric) initial pressures. With the recent interest in hydrogen economy, measurement of this dynamic parameter at high initial pressure condition is desirable to assess the potential detonation hazards of high pressure storage facilities of hydrogen $[8,9]$.

The detonation sensitivity behavior of hydrogen mixtures at elevated initial pressure can differ from that of other hydrocarbon mixtures due to the unique characteristics in hydrogen oxidation kinetics and the (extended) second explosion limit effects [10]. With increasing pressure, the chain-termination effect by the three-body termination reaction $\mathrm{H}+\mathrm{O}_{2}+\mathrm{M} \rightarrow$ $\mathrm{HO}_{2}+\mathrm{M}$ (R2) becomes significant and, thus, limits radical explosion by the two-body branching reaction $\mathrm{H}+\mathrm{O}_{2} \rightarrow \mathrm{O}+\mathrm{OH}$ (R1) [11]. The explosion sensitivity can be significantly decreased due to the explosion limit when the pressure is increased to a critical value. Although simple theoretical analysis based on chemical kinetics had indicated that above some critical pressure value, the required energy for direct initiation in hydrogen mixture increases gradually with increasing initial pressure [9], nevertheless, this phenomenon has not been verified experimentally and indeed only very few data on the critical energy of direct initiation at high-pressure conditions are available in literature. 
The aim of this paper is to evaluate detonation sensitivity of stoichiometric $\mathrm{H}_{2}-\mathrm{O}_{2}$ mixture at high initial pressure by measuring critical energy for direct initiation of spherical detonation. The experimental setup and protocol must be carefully designed with extra safety precautions due to the dangerous nature of this experiment with high voltage and high pressure conditions. Experiments are carried out in a spherical bomb and detonation initiation results from a high voltage capacitor spark discharge. One of the challenges in measuring critical direct initiation energy is to estimate the actual amount deposited into the mixture for initiation from the spark discharge. Therefore, various methods (i.e. the calorimeter method and current method) are explored and compared in this study to yield a good estimate of the correct amount of energy deposited into the mixture for initiating a detonation. These results shall be useful to understand the effect of chemical kinetics on hydrogen detonation sensitivity at high initial pressures and to develop a proper correlation model for safety assessment at the extreme conditions environment.

\section{EXPERIMENTAL SETUP}

The experiment is carried out in high pressure spherical explosion chamber 8” in diameter and 2" in wall thickness. A simple schematic and a picture of the experimental apparatus are given in Fig. 1. The chamber is connected to the gas manifold panel through a ball valve. The sphere is initially evacuated to at least $80 \mathrm{~Pa}$. The sphere is then incrementally filled through

the ball valve to prepare a mixture of hydrogen and oxygen at various test pressures. The explosive mixture is prepared directly inside the combustion chamber by the method of partial pressures. After the filling, the gases are allowed to mix in the chamber for at least 1 hour in order to ensure homogeneity. A heating element is placed at the bottom of the chamber to 
promote mixing through convection. A PCB piezoelectric pressure transducer is mounted in the wall of the chamber to measure the blast pressure and the time of arrival of the detonation front. The pressure record is used to determine if there is a successful initiation or not. The ignition system consists of a high voltage power supply, capacitor bank (Maxwell Series S/SS 0.02 to $2 \mu \mathrm{f})$, a three electrode gap-switch (GP-22B, GP-41B), a trigger module (TM-11A) and a slender coaxial electrode mounted on top of the explosion sphere. At the end of this slender electrode there is a $3.5 \mathrm{~mm}$ spark gap through which the energy is delivered inside the chamber through the ignition circuit.

\section{ENERGY ESTIMATION FROM THE HIGH-VOLTAGE CAPACITOR DISCHARGE}

\subsection{Current measurement method}

The energy deposited into the mixture is obtained using the current measurement method [12]. As seen in the ignition circuit diagram (Fig. 2), the energy deposited inside the sphere comes from the discharge of the high voltage capacitor bank. Total energy stored in the capacitors $\left(E_{T}=C V^{2} / 2\right)$ could be used to estimate the energy required to detonate the hydrogen-oxygen mixture. However this estimate will only give the order of magnitude approximation to the actual energy deposited in the sphere since it does not take into account energy loss in circuit components and loss via ohmic dissipation. Therefore, the actual energy

deposited inside the mixture is found by integrating the square of current function $i(t)^{2}$ multiplied by the spark resistance $\left(R_{\mathrm{s}}\right): E_{s}=\int_{0}^{\infty} i^{2} R_{s} d t$. The ignition circuit in Fig. 2 can be simplified to an equivalent RLC circuit shown in Fig. 3.

Thus for a damped oscillatory discharge the current can be described as: $i(t)=A e^{-\alpha \cdot t} \sin (\omega t)$. Attenuation factor is equal to $\alpha=R_{\text {total }} / 2 L_{\text {total }}$ and frequency of the 
discharge is equal to $\omega=\sqrt{1 /(L C)_{\text {total }}-(R / 2 L)_{\text {total }}^{2}}=\omega_{n} \sqrt{1-\varsigma^{2}}$, where $\omega_{n}$ is the natural frequency of the undamped discharge and $\zeta$ is the damping factor. The total circuit resistance $\left(R_{\text {total }}=R_{\text {circuit }}+R_{\mathrm{S}}\right)$ is a combination of the resistance of all the circuit components $R_{\text {circuit }}$ and igniter spark gap resistance $R_{\mathrm{s}}$. To estimate the energy $E_{\mathrm{s}}$ deposited into the mixture from a trace of the current function on the oscilloscope, the following procedure is outlined:

1. Natural frequency $\omega_{n}$ is determined using $\omega$ and $\zeta$ found from the current trace.

2. Total circuit inductance is found from $L_{\text {total }}=1 / \omega_{n}^{2} C_{\text {total }}$

3. Total circuit resistance is found as follows: $R_{\text {total }}=2 L_{\text {total }} \alpha$

4. Spark resistance $R_{\mathrm{S}}$ is determined by subtracting $R_{\text {total }}=R_{\text {circuit }}$ when the spark gap is shorted out $R_{\mathrm{s}} \approx 0$ from $R_{\text {total }}=R_{\text {circuit }}+R_{\mathrm{s}}$ when the spark is not shorted. Current traces of the two cases are shown in Fig. 4.

5. Total energy deposited into the mixture is found by numerically integrating the square of the current multiplied by spark resistance: $E_{s}=\int_{0}^{\infty} i^{2} R_{s} d t$

From previous study [12], it is shown that only the energy deposited in the first $1 / 4$ of the discharge cycle is responsible for detonating the mixture. The detonation velocity produced by the full discharge and $1 / 4$ discharge are identical [12] and most pulse energy is consumed in the first $1 / 4$ cycle based on emission intensity from the spark [13]. Hence, the detonation initiation energy obtained from each experiment should be described by the amount calculated from the first $1 / 4$ discharge current cycle. 


\subsection{Calorimeter Method}

Another technique that is being considered is the calorimeter method. It can serve as an alternative energy measurement method in order to verify results obtained from the current measurement method. The idea is that the energy deposited into the mixture is estimated by measuring the change in static pressure of air in a fixed volume container. Since the density in a fixed volume container remains constant the energy can be calculated using ideal gas law:

$$
E_{s}=\Delta Q=m_{\text {air }} \cdot C_{v} \cdot\left(T-T_{\text {initial }}\right)=m_{\text {air }} \cdot c_{v} \cdot T_{\text {initial }} \cdot\left(\frac{\left(P_{\text {initial }}+\Delta P\right)}{P_{\text {initial }}}-1\right)
$$

The results are presented in Fig. 5. The total stored energy in the capacitor $\left(E_{\text {total }}=1 / 2 C V^{2}\right)$ is plotted on the $x$-axis. The total spark energy obtained from the current measurement analysis normalized by the calorimetric measurement of energy is plotted on the $y$-axis. As seen in Fig. 5, the total spark energy is about $40 \%$ higher than the energy measured from the static pressure rise. One of the reasons for this discrepancy can be attributed to the heat loss in the calorimeter. Moreover ideal gas law assumes constant value for the heat capacity of the gas and does not account for variability of the heat capacity in the plasma channel of the spark. Thus based on the first pass calculation the spark energy measurement is in agreement with the calorimeter method within 40\%. A more detailed ideal gas law analysis taking into account all the associated losses will lead to improved accuracy estimation.

\section{RESULTS AND DISCUSSION}

In order to minimize the number of shots and iterations in the direct initiation experiments, the theoretical model proposed by Lee et al. [14, 15], linking the initiation energy and the detonation cell size, is used due to its simplicity to obtain some preliminary estimates on the critical energy values. The model is based on the idea that a minimum surface energy is 
required before a planar wave can evolve into a spherical wave without being quenched by expansion waves coming from the boundaries of the detonation wave itself. The link is therefore established based on the minimum surface energy of the critical tube to the surface area of the critical size of the minimum detonation kernel [15]. The surface energy contained in the point blast initiated spherical detonation wave at the time when the wave has decayed to the CJ state is equivalent to the energy in the planar detonation wave in the critical tube diameter. Hence equating the minimum surface areas of both waves at criticality:

$$
4 \pi R^{* 2}=\left(\frac{\pi}{4}\right) d_{c}^{2}
$$

yields a kernel radius $R^{*}=1 / 4 d_{\mathrm{c}}$. From strong blast wave theory, the blast wave energy is given by:

$$
E_{c}=4 \pi I \gamma p_{o} M_{s}^{2} R_{s}^{3}
$$

which for $R_{\mathrm{S}}=R^{*}$ at $M_{\mathrm{s}}=M_{\mathrm{CJ}}$ and with the empirical correlation of critical tube diameter $d_{\mathrm{c}}=$ $13 \lambda$, one obtains:

$$
E_{c}=4 \pi \gamma p_{o} M_{C J}^{2} I\left(\frac{13 \lambda}{4}\right)^{3}=\frac{2197}{16} \pi \rho_{o} V_{C J}^{2} I \lambda^{3}
$$

where $\rho_{0}$ is the initial density of the mixture, $V_{C J}$ the CJ detonation velocity, $\lambda$ the cell size and $I$ is a numerical constant (for $\gamma=1.4, I=0.423$ ) [16]. The characteristic cell size $\lambda$ for the hydrogen-oxygen and methane-oxygen mixtures at different initial pressures are estimated using a correlation model by $\mathrm{Ng}$ et al. $[8,9,17]$ and is shown in Fig. 6 , together with some experimental data obtained from the Caltech Detonation Database [18]. This model takes common form of $\lambda=A(\chi) \cdot \Delta$ where $\Delta$ is the characteristic ZND induction zone length which can be determined from chemical kinetics calculation $[19,20]$. However, $A(\chi)$ here represents a proportionality factor and is a function of a stability parameter $\chi$ [21]. This correlation 
model is validated against different cell size data and is shown to provide a good cell size estimates for hydrogen and other common hydrocarbon mixtures [8, 9, 17]. Details of this correlation model can be found in $\mathrm{Ng} \&$ Lee [8]. It is important to clarify that it is not the scope of this paper to survey different existing theories for direct initiation of detonation or to develop new predictive models. The main focus of this work is to report new accurate data of critical initiation energies for high pressure hydrogen-oxygen mixtures, results widely missing in the literature; and the theory used in this study is to provide some first guidance and estimates to prepare the experiments.

To classify a detonation as showing either a failure or successful direct initiation, the pressure can be analyzed using the following criteria. Figure 7 shows the pressure traces for a successful direct initiation and unsuccessful initiation of a spherical detonation in the stoichiometric $\mathrm{H}_{2}-\mathrm{O}_{2}$ mixture at an initial pressure of $p_{0}=300 \mathrm{kPa}$. For successful initiation, a large pressure rise occurs about $71 \mu$ sec indicating a detonation. For a lower initiation energy (i.e. spark energy $1 / 4$ cycle), no significant pressure rise happens until $703 \mu$ sec at which point the mixture is initiated by auto-ignition due to the shock reflection from the chamber wall. In such case, no direct initiation occurs. Table 1 summarizes the details of the experimental results and parameters for successful direct initiation and unsuccessful initiation.

Theoretical critical energy values obtained using Lee’s surface energy model as well as present experimental results are plotted for various initial pressures in Fig. 8. Note that for each initial pressure, at least 4 to 5 shots were performed with small energy increment to determine and narrow the range of critical initial energy for direct initiation. However, only the interval limits for GO/NO GO are shown in the Fig. 8. Critical energy estimates by Zitoun et al. [7] are also included in the plot. In the work of Zitoun et al. [7] the energy was 
calculated using strong point source explosion theory [22] from shock pressure measurements at a given shock radius. The critical energy determined in this way was reported to be accurate with $\pm 50 \%$. As seen in Fig. 8 experimental results follow the same trend as predicted by Lee’s surface energy theoretical model. At elevated initial pressures the critical energy reaches a relatively constant value below which a detonation cannot be directly initiated. This plateau can be attributed to second explosion limit. The second explosion limit results from the competition between the branching process $\mathrm{H}+\mathrm{O}_{2} \rightarrow \mathrm{O}+\mathrm{OH}$ (R1) and the three-body termination process $\mathrm{H}+\mathrm{O}_{2}+\mathrm{M} \rightarrow \mathrm{HO}_{2}+\mathrm{M}$ (R2) as pressure increases. Using steady-state approximation the (extended) second explosion limit condition can be obtained from the kinetic rate relation $2 k_{1}=k_{2} \cdot[M]$, where $[M]$ is the third body concentration. As shown by Belles [23], it is convenient to express the branched-chain explosion kinetics condition in terms of shock strength by using the standard normal shock wave relationship. This gives a critical Mach number satisfying the kinetic requirement for explosion. Figure 9 shows the variation of the critical Mach number associated with the extended second explosion limit (using the kinetic information from [24]) and the detonation Mach number as a function of initial pressure for stoichiometric hydrogen-oxygen mixtures. At some critical threshold, there is a crossover point between these two Mach numbers. Above this critical point $M \sim 5.57$, the temperature behind the leading front of the ZND detonation structure is below the crossover temperature $T_{\mathrm{B}}$, at which the chain-termination reaction dominates over the chain-branching reaction, as predicted by the extended second-limit criterion. This critical point corresponds to an initial pressure about $18 \mathrm{~atm}$. The variation in critical energy appears to agree with this observation. For an initial pressure above 18 atm, the present experimental values appear to show that the critical energy may reach a plateau. This is in good agreement with the 
theoretical prediction obtained in previous studies [9] that the energy reaches a minimum when the initial pressure passed the critical pressures and slightly increases for increasing higher initial pressures.

In this study, the (extended) second explosion limit is used as a chemical kinetic consideration on the detonation sensitivity. The effect should also have a direct influence on the reaction zone structure and hence, the characteristics in the multi-headed detonation front. Above the critical initial pressure defining the "extended" second explosion limit, the slowbranching reaction can prolong the induction zone [25], which shall be directly related to the cell size variation and the detonation front instability as shown for example by Liang and Bauwens [26].

To gauge the decrease in sensitivity of stoichiometric $\mathrm{H}_{2}-\mathrm{O}_{2}$ mixture at high initial pressure, the critical energy of this mixture obtained from the present experiment is compared to that for stoichiometric $\mathrm{CH}_{4}-\mathrm{O}_{2}$ mixture estimated using the theoretical model by Lee et al. [14]. Thus, as seen in Fig. 10 the critical energy of stoichiometric $\mathrm{H}_{2}-\mathrm{O}_{2}$ mixture at elevated initial pressure becomes comparable to that of stoichiometric $\mathrm{CH}_{4}-\mathrm{O}_{2}$ mixture. Equivalently, it may conclude that hydrogen-oxygen mixture is not more sensitive than any typical hydrocarbon mixture (such as $\mathrm{CH}_{4}-\mathrm{O}_{2}$ ) at elevated initial pressure.

\section{CONCLUDING REMARKS}

This paper presented new results of critical direct detonation initiation energy in high pressure stoichiometric hydrogen-oxygen mixture. Direct initiation is achieved via a high voltage capacitor discharge and a detailed explanation on how to obtain accurately the energy 
discharge using both the current measurement and calorimeter method is provided in this paper. This new reliable set of results will be useful in future to develop theory for direct initiation of detonation and validate models for safety assessment.

The second objective of this study is to obtain experimental data characterizing detonation sensitivity at high initial pressure conditions, where experimental data are lacking. In particular, the effect of second explosion limit on the detonation sensitivity of hydrogen-air mixture is examined. It is found that at elevated initial pressure, the hydrogen mixture becomes less sensitive with further increase in initial pressure. The sensitivity of stoichiometric $\mathrm{H}_{2}-\mathrm{O}_{2}$ mixture at elevated initial pressure becomes comparable to that of stoichiometric $\mathrm{CH}_{4}-\mathrm{O}_{2}$ mixture. It is thus demonstrated that the second explosion limit effect plays a significant role leading to slow branching reactions above a critical pressure threshold.

\section{ACKNOWLEDGMENT}

This work is supported by the Natural Sciences and Engineering Research Council of Canada (NSERC). 


\section{REFERENCES}

[1] J.H.S. Lee, A.J. Higgins, Phil. Trans. R. Soc. Lond. A 357 (1999) 3503-3521.

[2] H. Matsui, J.H.S. Lee, Proc. Combust. Inst. 17 (1978) 1269.

[3] E.L. Litchfield, M.H. Hay, D.R. Forshey, Proc. Combust. Inst. 19 (1962) 282-286.

[4] W.B. Benedick, C.M. Guirao, R. Knystautas, J.H.S. Lee, Prog. Astronaut. Aeronaut. 106 (1986) 181-202.

[5] R. Atkinson, D.C. Bull, P.J. Shuff, Combust. Flame 39(3) (1980) 287-300.

[6] C.M. Guirao, R. Knystautas, J. Lee, W. Benedick, M. Berman, Proc. Combust. Inst. 19 (1982) 583-590.

[7] R. Zitoun, D. Desbordes, C. Guerraud, B. Deshaies, Shock Waves 4(6) (1995) 331-337.

[8] H.D. Ng, J.H.S. Lee, J. Loss Prev. Proc. Ind. 21 (2008) 136-146.

[9] H.D. Ng, Y. Ju, J.H.S. Lee, Int. J. Hydrogen Energy 32 (2007) 93-99.

[10] D.W. Stamps, S.R. Tieszen, Combust. Flame 83 (1991) 353-364.

[11] B. Lewis, G. von Elbe, Combustion, Flames, and Explosions of Gases, Academic Press, New York, 1951.

[12] R. Knystautas, J.H.S. Lee, Combust. Flame 27 (1976) 221-228.

[13] V.P. Zhukov, A.E. Rakitin, A.Yu. Starikovskii, J. Prop. Power 24 (2008) 88-93.

[14] J.H.S. Lee, R. Knystautas, C.M. Guirao, in: J.H.S. Lee, C.M. Guirao (Eds.), Fuel-Air Explosions, University of Waterloo Press, 1982, p. 157.

[15] J.H.S. Lee, R. Ramamurthi, Combust. Flame 27(3) (1976) 331-340.

[16] G.G. Bach, J.H.S. Lee, AIAA J. 8 (1970) 271-275.

[17] H.D. Ng, The Effect of Chemical Reaction Kinetics on the Structure of Gaseous Detonations, PhD thesis, McGill University, Canada, 2005.

[18] M. Kaneshige, J.E. Shepherd, Detonation database, GALCIT Technical Report FM97-8, http://www.galcit.caltech.edu/detn_db/html/, 1997. 
[19] J.E. Shepherd, Prog. Astronaut. Aeronaut. 106 (1986) 263-293

[20] C.K. Westbrook, P.A. Urtiew, Proc. Combust. Inst. 19 (1982) 615-623.

[21] H.D. Ng, M.I. Radulescu, A.J. Higgins, N. Nikiforakis, J.H.S. Lee, Combust. Theory Model. 9(3) (2005) 385-401.

[22] V.P. Korobeinikov, Problems of Point-Blast Theory, American Institute of Physics, New York, 1991.

[23] F.E. Belles, Proc. Combust. Inst. 7 (1959) 745-751.

[24] J. Li, Z.W. Zhao, A. Kazakov and F.L. Dryer, Int. J. Chem. Kinet. 36 (2004) 566-575.

[25] T. Lu, C.K. Law and Y. Ju, J. Prop. Power 19 (2003) 901-907.

[26] Z. Liang, L. Bauwens, Shock Waves 15 (2006) 247-257. 


\section{TABLE CAPTIONS}

Table 1. Results for successful direct initiation and unsuccessful initiation for stoichiometric hydrogen-oxygen mixture at $p_{0}=300 \mathrm{kPa}$. 


\section{FIGURE CAPTIONS}

Figure 1. Experimental setup.

Figure 2. Ignition circuit components.

Figure 3. Equivalent RLC circuit diagram.

Figure 4. Discharge current through shorted and open spark gap in stoichiometric $\mathrm{H}_{2}-\mathrm{O}_{2}$ mixture. $\left(P_{0}=3\right.$ atm; $\left.V_{0}=14 \mathrm{kV} ; C_{\text {total }}=0.1983 \mu \mathrm{F}\right)$.

Figure 5. Comparison between spark energy vs. energy measured in a calorimeter.

Figure 6. Cell size estimation using the model by $\mathrm{Ng}$ et al. [9, 17]. Experimental data are obtained from the Caltech Detonation Database [18].

Figure 7. Pressure traces for the successful direct initiation and unsuccessful initiation of a spherical detonation in stoichiometric hydrogen-oxygen mixture at $300 \mathrm{kPa}$.

Figure 8. Critical energy calculated using the simple relationship and measured value from experiments as well as results by Zitoun et al. [7].

Figure 9. The critical Mach number corresponding to the second explosion limit criterion and detonation Mach number as a function of initial pressure for the stoichiometric hydrogen-oxygen mixture.

Figure 10. Comparison of critical energy for direct initiation of detonation in stoichiometric $\mathrm{H}_{2}-\mathrm{O}_{2}$ and stoichiometric $\mathrm{CH}_{4}-\mathrm{O}_{2}$ mixtures. 


\begin{tabular}{|c|c|c|c|c|c|c|c|c|}
\hline & & & & \multicolumn{3}{|c|}{ Spark } & & \multirow[b]{2}{*}{$\begin{array}{l}\text { Direct } \\
\text { initiation }\end{array}$} \\
\hline $\begin{array}{l}E \\
0.5 \mathrm{CV} \wedge 2\end{array}$ & $\begin{array}{l}V_{\mathbf{o}} \\
(\mathrm{kV})\end{array}$ & $\begin{array}{l}C_{\text {circuit }} \\
(\mu \mathrm{F})\end{array}$ & $\begin{array}{l}R_{\text {circuit }} \\
(\boldsymbol{\Omega})\end{array}$ & $\begin{array}{l}R_{\text {spark }} \\
(\boldsymbol{\Omega})\end{array}$ & $\begin{array}{l}E_{1 / 4 \text { cycle }} \\
\text { (J) }\end{array}$ & $\begin{array}{l}E_{\text {total }} \\
\text { (J) }\end{array}$ & $\% V_{C J}$ & \\
\hline 28.7 & 17 & 0.1983 & 0.2374 & 0.0424 & 0.7104 & 4.0755 & 91 & yes \\
\hline 35.8 & 14 & 0.1983 & 0.2444 & 0.0271 & 0.6574 & 3.5586 & 16 & no \\
\hline
\end{tabular}

Table 1. Results for successful direct initiation and unsuccessful initiation for stoichiometric hydrogen-oxygen mixture at $p_{0}=300 \mathrm{kPa}$. 


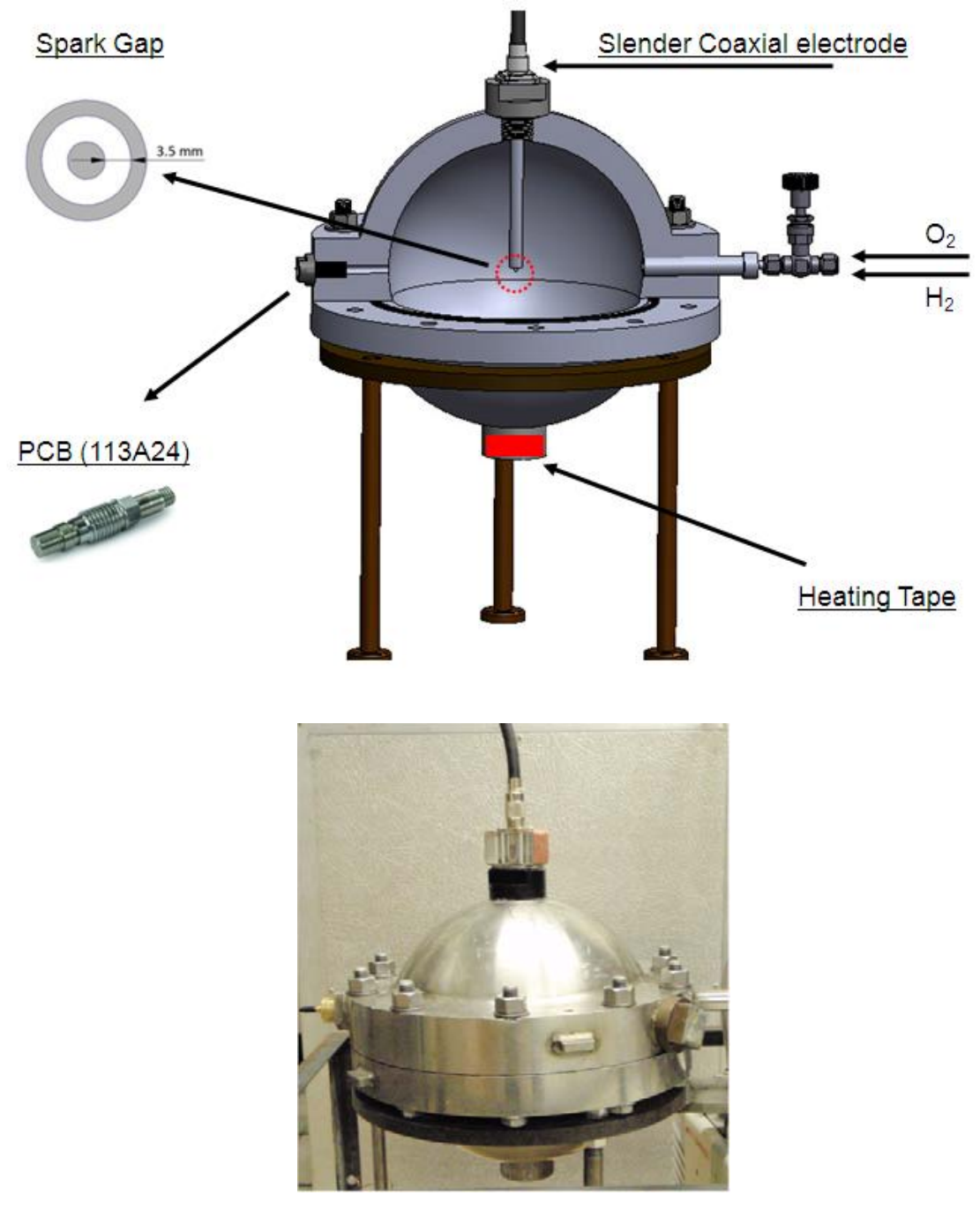




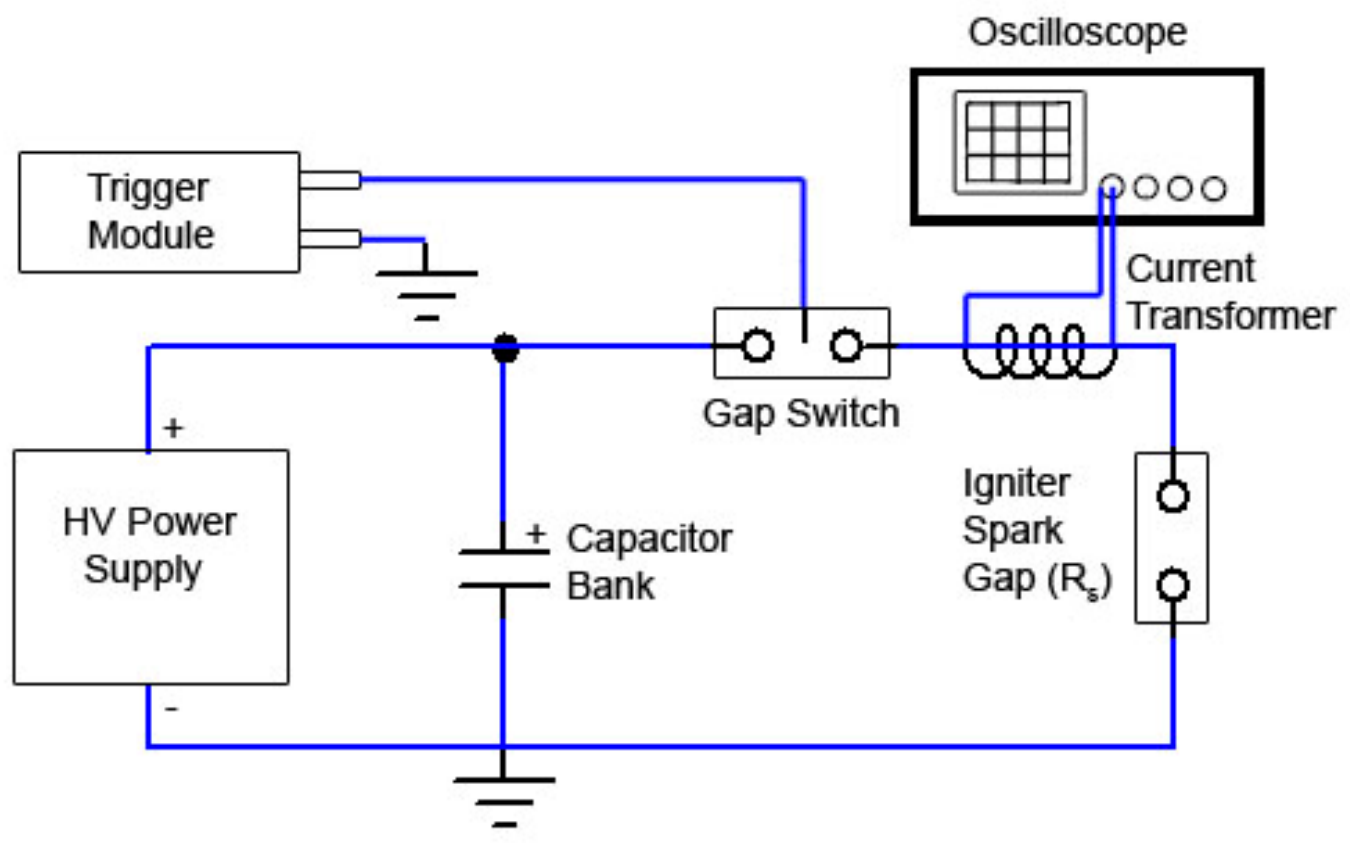




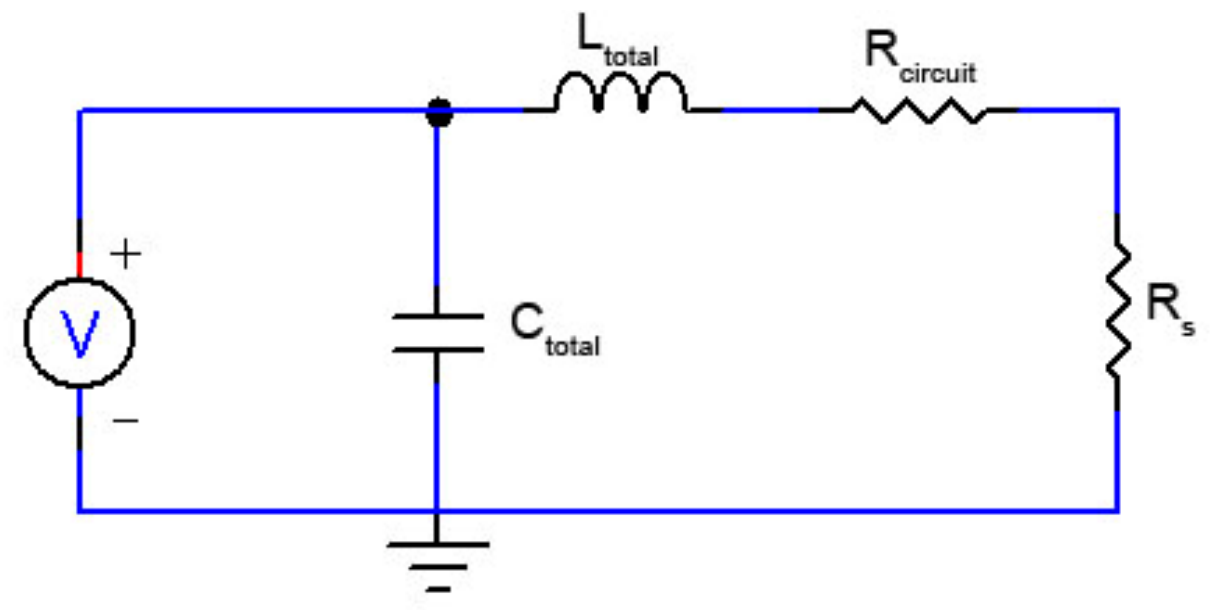




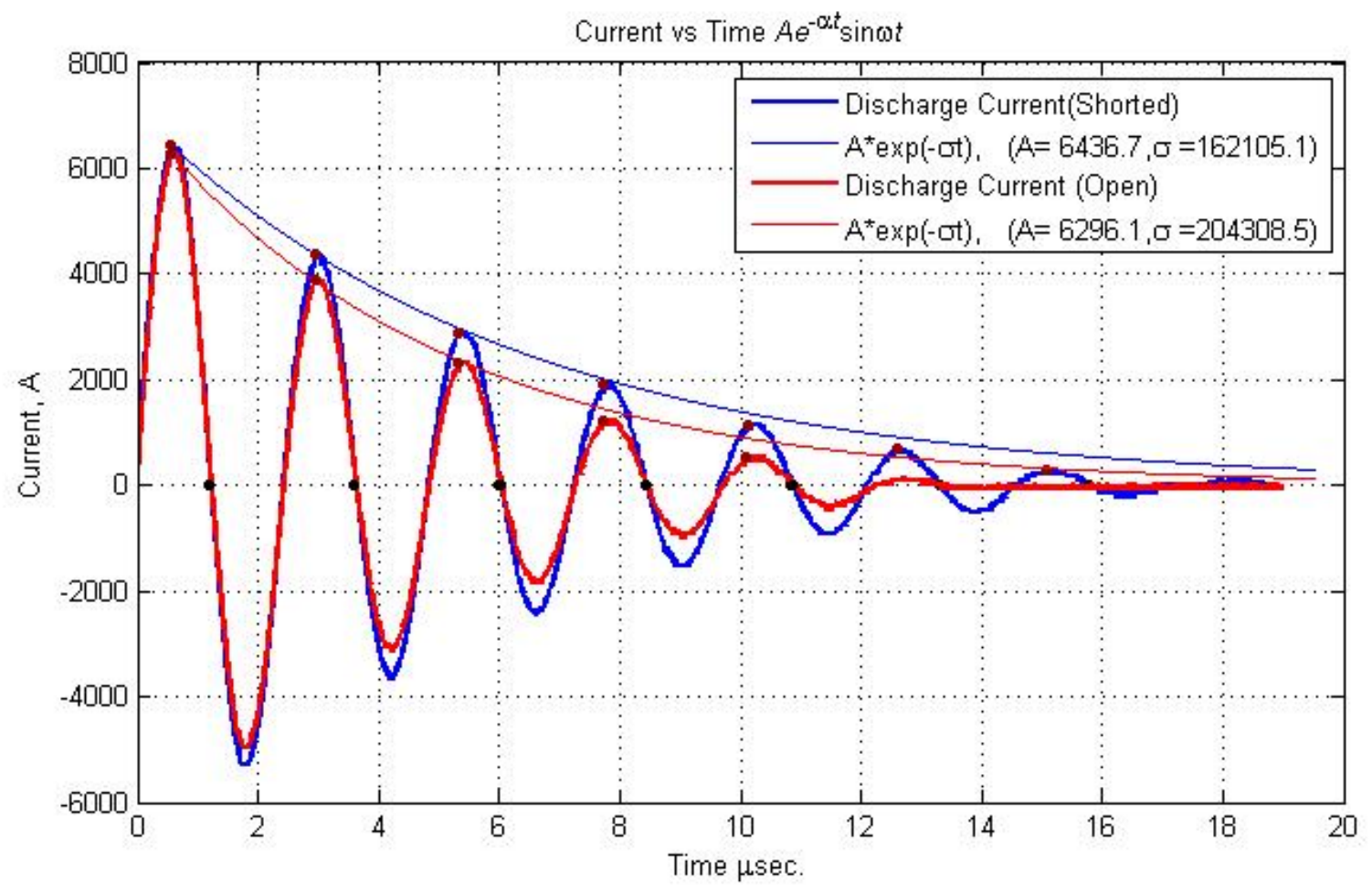




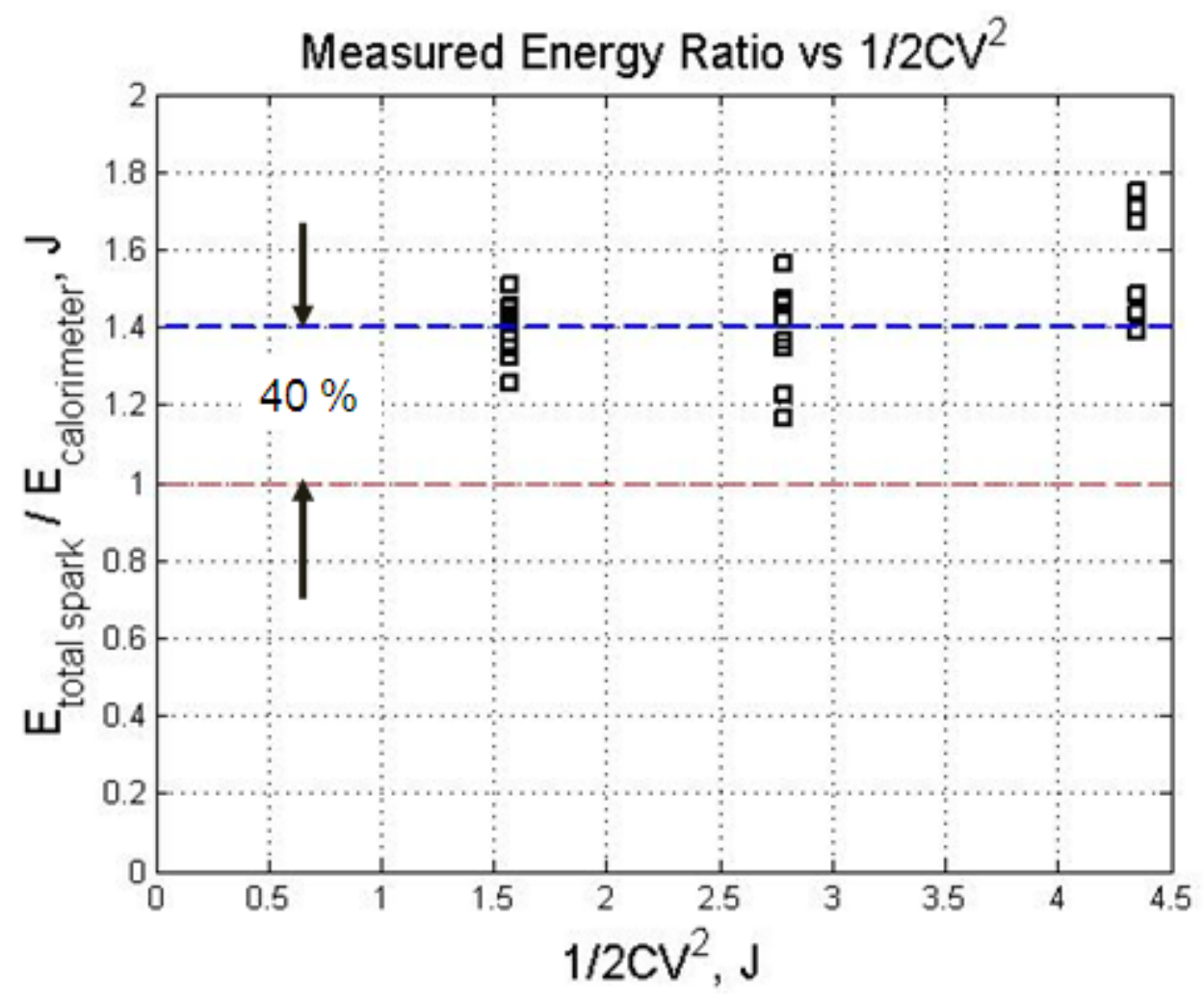




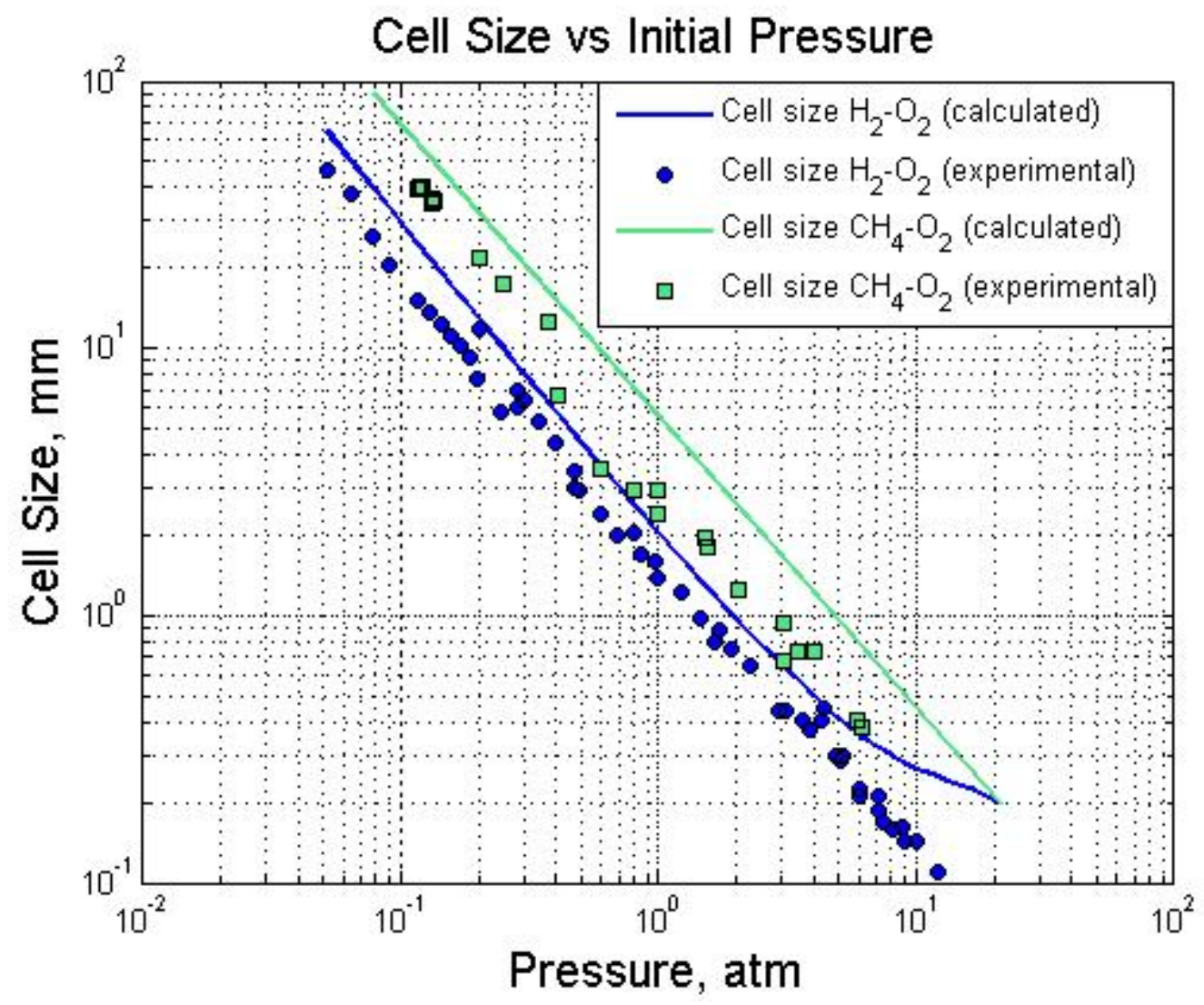




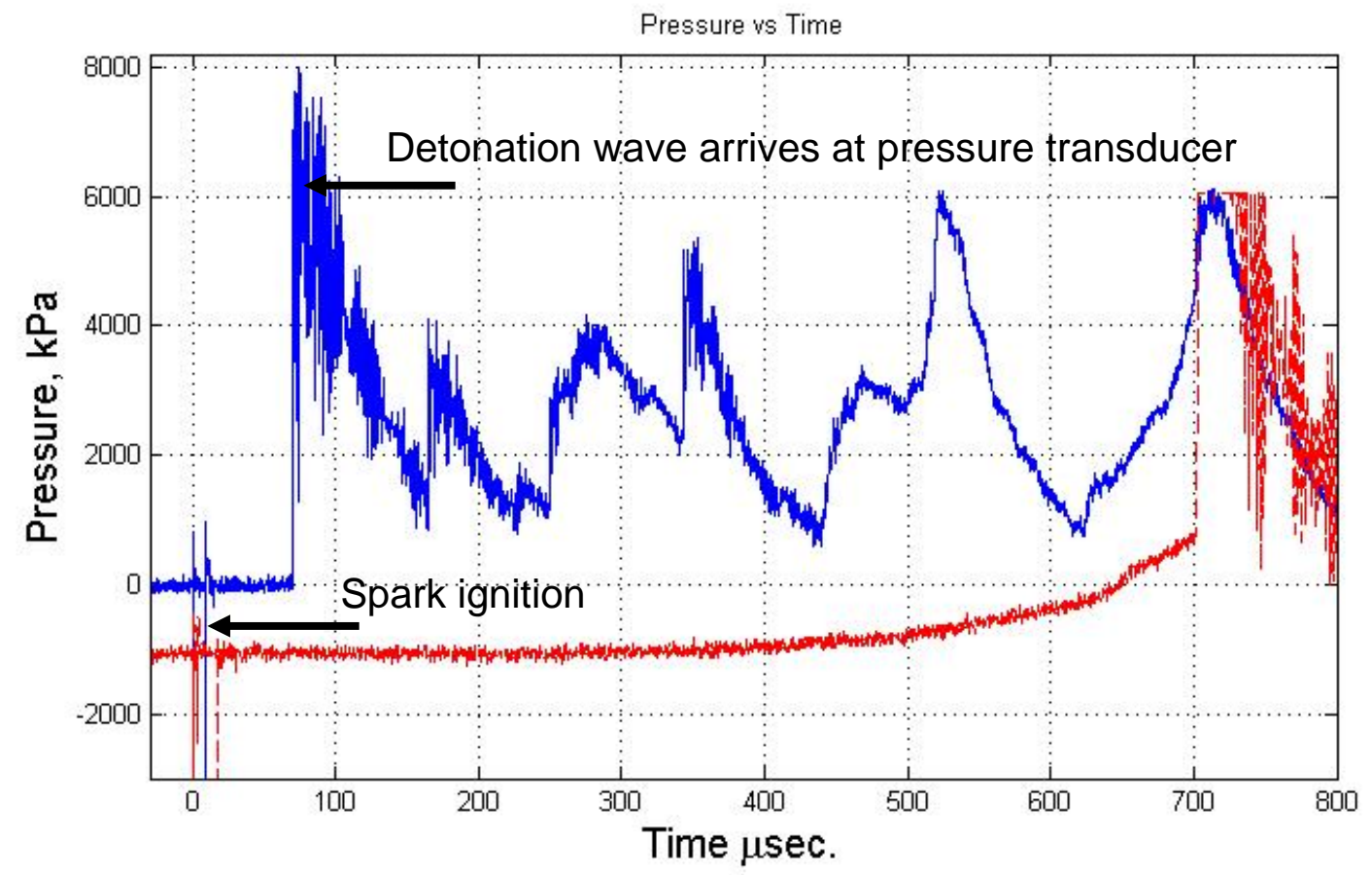




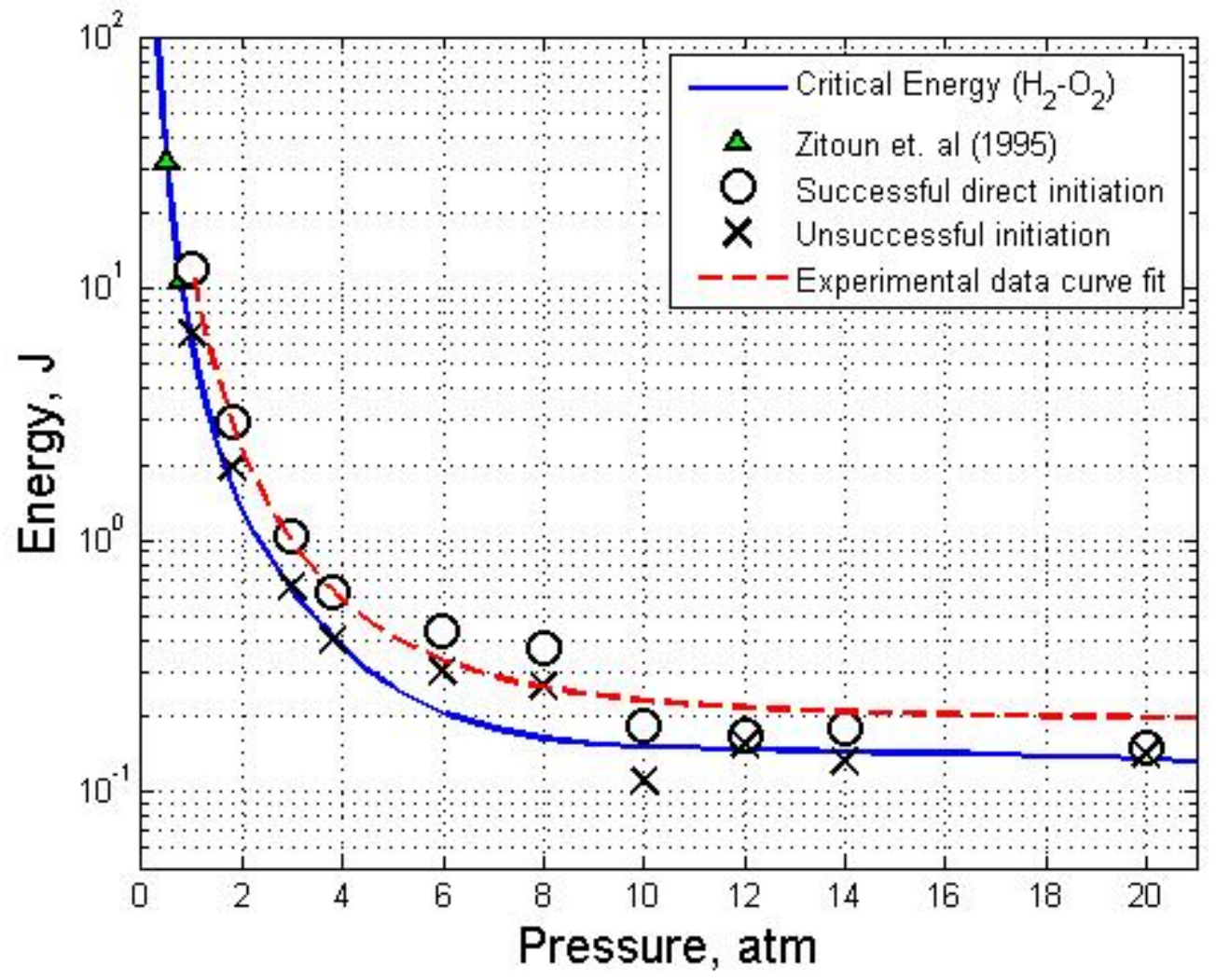




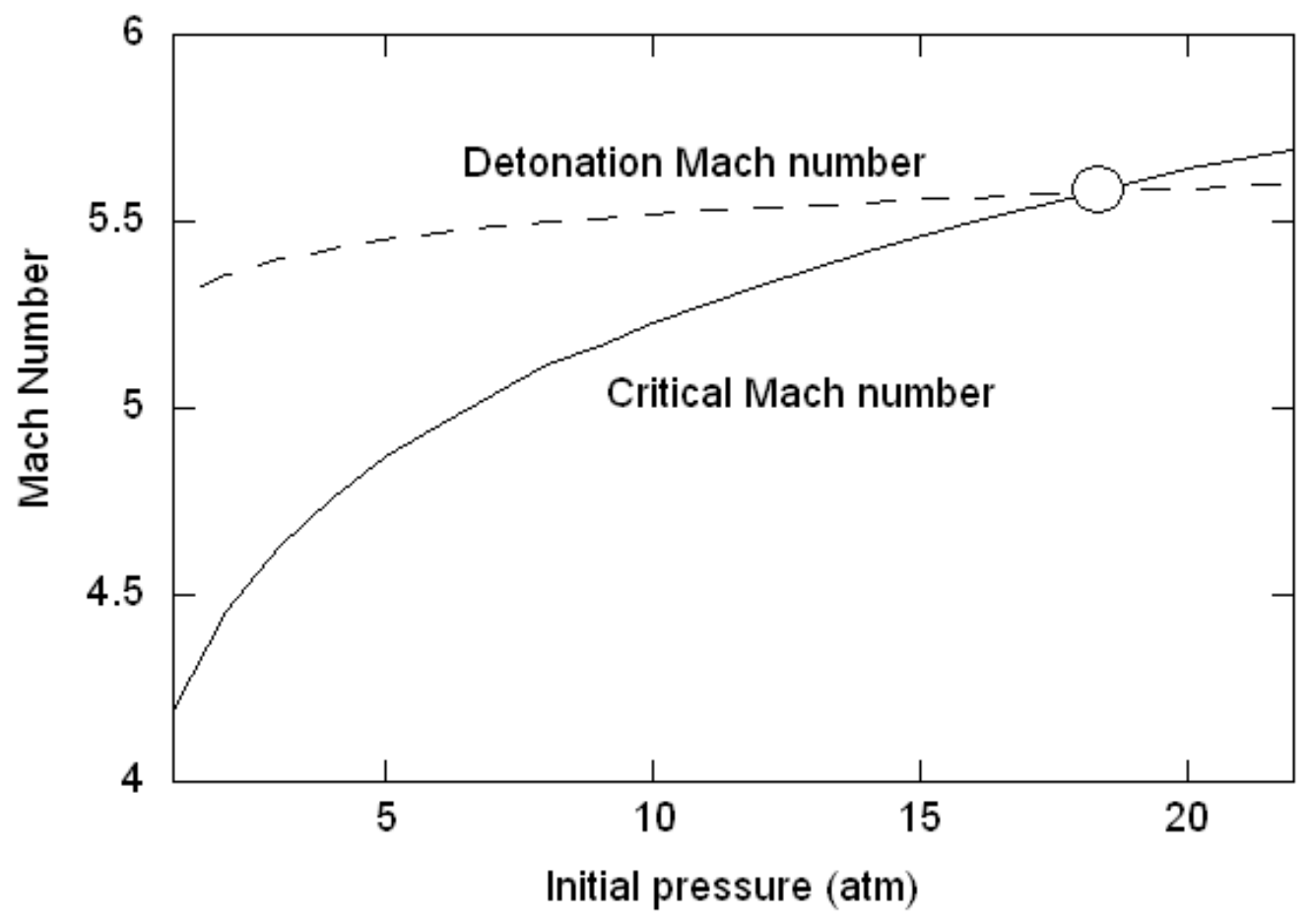




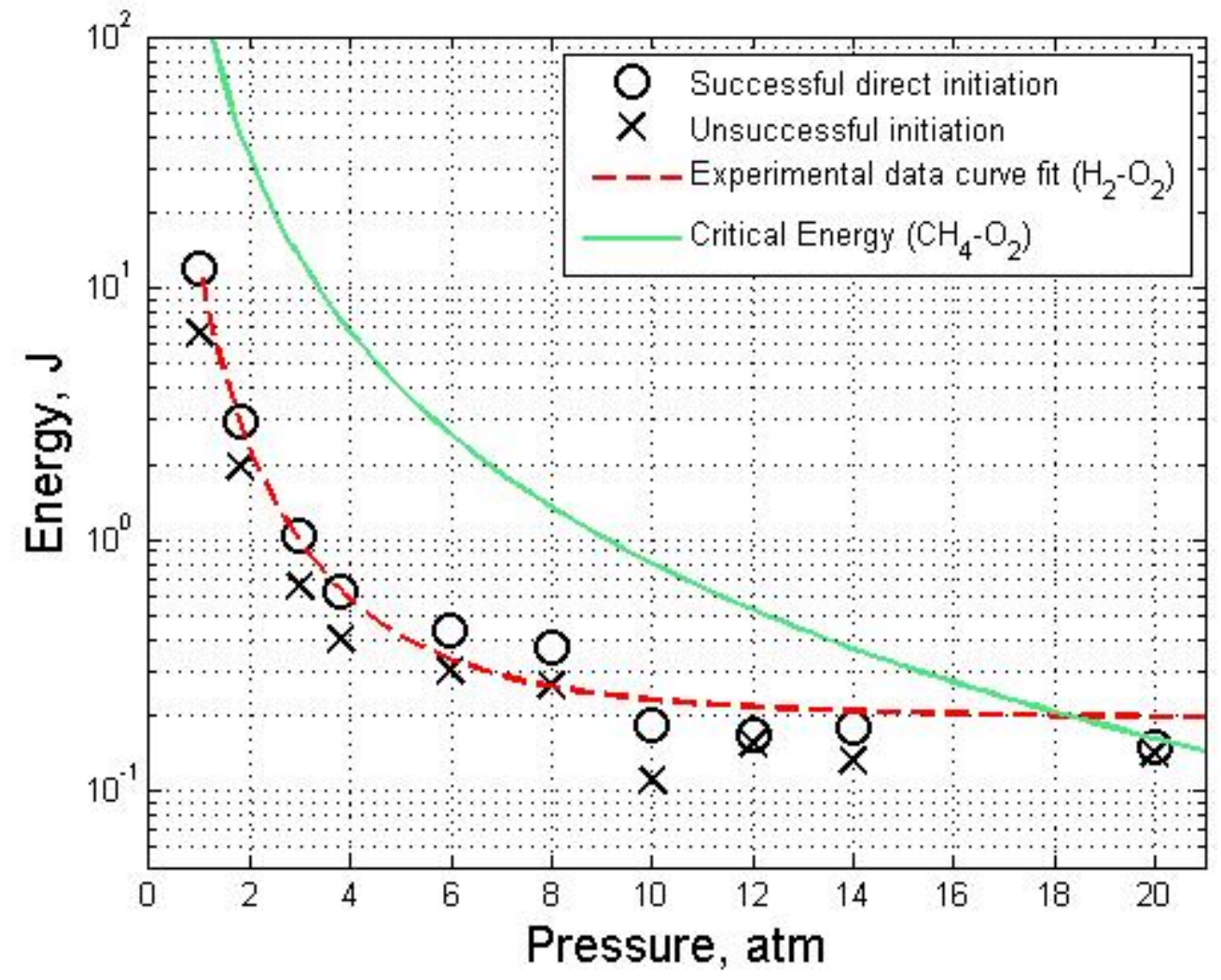

